TOKYO J. MATH

VOL. 28, No. 2, 2005

\title{
The Number of Vogel Operations to Deform a Link Diagram to a Closed Braid
}

\author{
Chuichiro HAYASHI and Hiroko SAEKI \\ Japan Women's University \\ (Communicated by K. Taniyama)
}

\begin{abstract}
Vogel showed that any oriented link diagram $D$ can be deformed to a closed braid by a finite sequence of Reidemeister II moves, each performed on two coherently oriented edges in a face of $D$ such that the edges are contained in distinct Seifert circles. We show that the number of such moves is constant for a given oriented link diagram, and does not depend on the sequence of moves. An easy way of calculating the number is given.
\end{abstract}

\section{Introduction}

J. W. Alexander showed in [1] that any oriented link diagram $D$ can be deformed to a closed braid. In [3], S. Yamada accomplished this by a sequence of certain operations on $D$, each of which does not change the number of the Seifert circles derived from $D$. P. Vogel showed in [2] that any oriented link diagram $D$ can be deformed to a closed braid by a finite sequence of a special kind of Reidemeister II moves, which he called elementary operations. The diagram $D$ can be regarded as a 4-valent graph, the vertices of which are the crossings. The edges of $D$ have orientations derived from that of $L$. Each elementary operation is performed in a face $f$ of $D$ on two edges $\alpha, \beta$ which are coherently oriented (i.e., their orientations agree with those induced from some orientation of $f$ ) and contained in distinct Seifert circles. See Figure 1. Such an operation does not change the number of Seifert circles. He called $(f, \alpha, \beta)$ an admissible triple. He showed that the number of operations in any such sequence is $\frac{(n+1)(n+2)}{2}-\chi(D)$ or smaller, where $n$ is the number of the Seifert circles. We recall later the definition of $\chi(D)$ which is a positive integer for any $D$, (and is not the Euler characteristics as in the usual notation in Topology).

We cite here the first two sentences in Remark 5-3 in [2]: "The number of elementary operations $T$ needed to transform a diagram $D$ in a closure of a braid is not completely clear. It depends on the sequence of admissible triples." However, we show in this paper that, in fact, it does not. The number of operations is determined only by $D$ and does not depend on the sequence. An easy way of calculating the precise number is given in the next section.

Received July 1, 2003; revised February 22, 2005

The first author was partially supported by Grant-in-Aid for Scientific Research (No. 15740047), Ministry of Education, Science and Culture. 

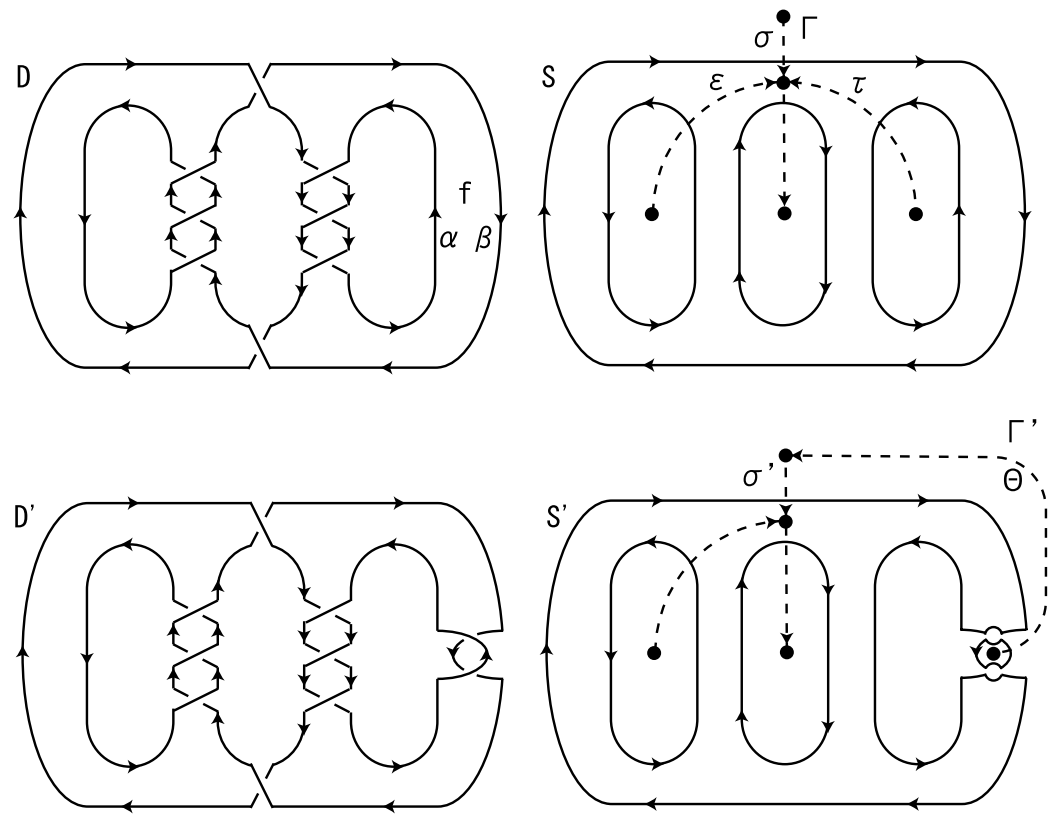

FIGURE 1. Elementary operation.

In the rest of this section, we recall the machinery of the proof in [2], and the definition of $\chi(D)$. We regard the diagram $D$ is on a 2-sphere $\Sigma$. Let $S$ be the union of Seifert circles derived from $D$. The circles of $S$ have orientations induced from that of $D$. Cutting the 2sphere $\Sigma$ along $S$, we obtain plural connected components, each of which is called a region of $S$. We form a graph $\Gamma$ associated to $S$. Every region of $S$ contains precisely one vertex of $\Gamma$ in its interior. Two vertices $v_{0}, v_{1}$ are connected by an edge $e$ if and only if the corresponding regions $f_{0}, f_{1}$ share a Seifert circle $C$ of $S$ in their boundaries. We place $e$ so that it intersects $S$ in a single transverse point in $C$, and orient it so that $v_{0}$ is the initial vertex and $v_{1}$ is the terminal vertex if $f_{0}$ is on the left hand side of $C$ and $f_{1}$ on right with respect to the orientations of $C$ and $\Sigma$. (In Figure 1, terminal vertices are furnished with arrow-heads.) Then $\Gamma$ is a tree with all its edges oriented.

A tree with the edges oriented is called a chain if either it consists of a single vertex and no edges, or it has precisely two isolated vertices (i.e., the vertices of valency 1) and all the edges are oriented coherently (i.e., each of the other vertices is the terminal vertex of a single edge and the initial vertex of a single edge). The diagram $D$ is a closed braid if and only if $\Gamma$ is a chain. $\chi(D)=\chi(\Gamma)$ is the number of the subtrees of $\Gamma$ which are chains.

Let $D^{\prime}$ be the link diagram obtained by an elementary operation on an admissible triple $(f, \alpha, \beta)$ of $D$. We can obtain the tree $\Gamma^{\prime}$ associated to $D^{\prime}$ from $\Gamma$ as below. Let $u$ be the vertex corresponding to the region of $S$ which contains the face $f$ of $D$. Let $\sigma$ and $\tau$ be the edges corresponding to the Seifert circles which contain $\alpha$ and $\beta$ respectively. Either $u$ is the 
initial vertex of $\sigma$ and $\tau$, or the terminal vertex of them. We consider the former case. (The latter case is similar, and is described in Figure 1.) We amalgamate the edges $\sigma$ and $\tau$ to an edge $\sigma^{\prime}$, and add a new edge $\theta$ so that the terminal vertex of $\sigma^{\prime}$ is the initial vertex of $\theta$ and that the terminal vertex of $\theta$ is isolated. The resulting graph is the desired $\Gamma^{\prime}$. Such a deformation on a tree with the edges oriented is called an elementary transformation, independently from link diagrams.

As mentioned in Remark 5-3 in [2], it is possible that we cannot perform an elementary operation corresponding to a given elementary transformation on a triple $(u, \sigma, \tau)$ of $\Gamma$. This happens when the Seifert circles corresponding to $\sigma$ and $\tau$ do not meet the same face of $D$. (For example, we cannot perform an elementary operation on $D$ corresponding the pair of the edges $\varepsilon$ and $\tau$ in Figure 1.) Note that the region of $S$ corresponding to $u$ may be divided to faces of $D$ by crossings of $D$. However, even in such a case, there is another pair of edges $\sigma^{\prime}$ and $\tau^{\prime}$ sharing the initial vertex $u$, such that we can perform a corresponding elementary operation on $D$.

\section{Definitions and results}

Let $\Gamma$ be a finite tree with all the edges are oriented. (We assume neither property (P) nor (P1) in [2].)

A connected subgraph of $\Gamma$ is called a path if either it consists of a single vertex and no edges or it has precisely two isolated vertices. A path may be a union of plural chains with orientaions of adjacent ones incoherent.

A height function $h$ on $\Gamma$ assigns an integer $h(v)$ to every vertex $v$ of $\Gamma$ so that $h(u)=$ $h(w)+1$ for every edge with the initial vertex $w$ and the terminal vertex $u$.

LEMma 2.1. (1) There exists a height function on $\Gamma$, and (2) for arbitrary two height functions $h_{1}$ and $h_{2}$, the difference $h_{1}(v)-h_{2}(v)$ is constant over all the vertices of $\Gamma$.

PROOF. If $\Gamma$ consists of a single vertex and no edges, this lemma is very clear.

We prove first (1) by induction on the number of edges of $\Gamma$. Suppose $\Gamma$ has one or more edges. Since $\Gamma$ is a tree, it has an isolated vertex $w$. Then $w$ is incident to a single edge $f$. Let $u$ be the other vertex of $f$. We obtain a tree $\Gamma^{\prime}$ from $\Gamma$ by removing $w$ and $f$. There is a height function $h^{\prime}$ on $\Gamma$ by the assumption of induction. If $w$ is the initial vertex of $f$, then we set $h(w)=h^{\prime}(u)-1$, and $h(v)=h^{\prime}(v)$ for the other vertices of $\Gamma$. If $w$ is the terminal vertex of $f$, then we set $h(w)=h^{\prime}(u)+1$, and $h(v)=h^{\prime}(v)$ for the other vertices of $\Gamma$. Thus we can obtain a height function $h$ on $\Gamma$.

We prove (2) by induction. Let $w, f, \Gamma^{\prime}$ be as above. For $i=1$ and 2 , let $h_{i}^{\prime}$ be the height function on $\Gamma^{\prime}$ obtained from the height function $h_{i}$ on $\Gamma$ by restricting it on $\Gamma^{\prime}$. Then $h_{1}^{\prime}(v)-h_{2}^{\prime}(v)$ is constant over all the vertices of $\Gamma^{\prime}$ by the assumption of induction. Hence $h_{1}(v)-h_{2}(v)$ is also constant over all the vertices of $\Gamma^{\prime}$. If $w$ is an initial vertex of $f$, then $h_{1}(w)-h_{2}(w)=\left(h_{1}(u)-1\right)-\left(h_{2}(u)-1\right)=h_{1}(u)-h_{2}(u)$. If $w$ is a terminal vertex of $f$, then $h_{1}(w)-h_{2}(w)=\left(h_{1}(u)+1\right)-\left(h_{2}(u)+1\right)=h_{1}(u)-h_{2}(u)$. This shows $(2)$. 


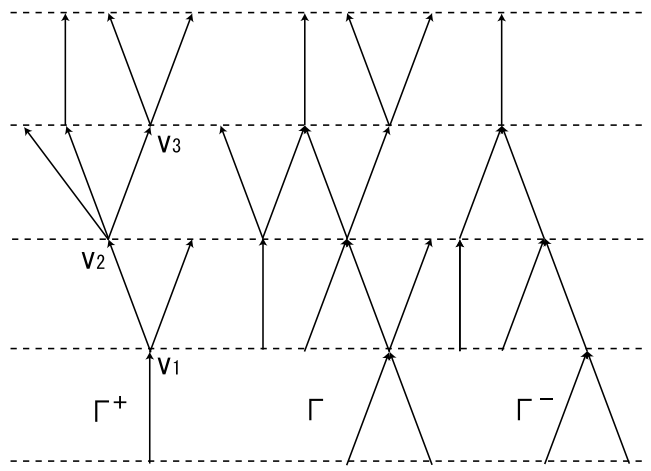

FIGURE 2. Formation of $\Gamma^{+}$and $\Gamma^{-}$.

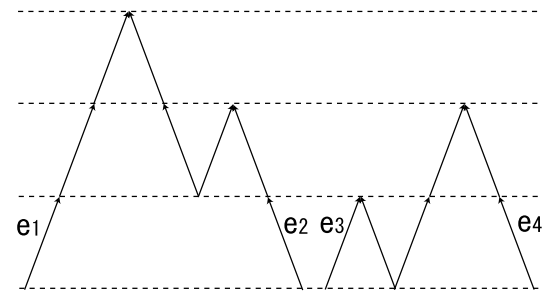

FIGURE 3. $e_{1}$ and $e_{2}$ are to be amalgamated.

A vertex is at the same height as (or higher than) another vertex with respect to $h_{1}$ if and only if it is so with respect to $h_{2}$ by the above lemma. We fix a height function $h$ on $\Gamma$.

A pair of edges $e_{1}$ and $e_{2}$ is called an upper branch pair if they share the initial vertex, which is called an upper branch point. Similarly we define a lower branch pair and a lower branch point.

We form a graph $\Gamma^{+}$from $\Gamma$ by amalgamating edges of $\Gamma$. See Figure 2. Two edges $e_{1}$ and $e_{2}$ of $\Gamma$ are amalgamated if and only if their terminal vertices $v_{1}, v_{2}$ are at the same height $h$ and the path connecting $v_{1}$ and $v_{2}$ in $\Gamma$ has no vertex at height lower than $h$. The path is uniquely determined by $v_{1}$ and $v_{2}$ because $\Gamma$ is a tree. In Figure $3 e_{1}$ and $e_{2}$ are to be amalgamated, while $e_{3}$ and $e_{4}$ are not. The vertices of $\Gamma^{+}$have height derived from those of $\Gamma$.

\section{Lemma 2.2. The graph $\Gamma^{+}$is a tree.}

Proof. Suppose, for a contradiction, that $\Gamma^{+}$contains a cycle. It contains no lower branch pair of edges because they should be amalgamated in $\Gamma^{+}$. Hence all the edges are oriented coherently on the cycle. Let $v_{0}, v_{1}, \cdots, v_{m-1}, v_{m}=v_{0}$ be the vertices of the cycle appearing in this order on it, so that $h\left(v_{0}\right)<h\left(v_{1}\right)<\cdots<h\left(v_{m-1}\right)<h\left(v_{m}\right)=h\left(v_{0}\right)$. This is a contradiction. 
Similarly we form $\Gamma^{-}$. We will show the lemmas below in the next section.

LEMMA 2.3. Let $\Gamma^{\prime}$ be a tree obtained by amalgamating a lower branch pair of edges of $\Gamma$. Then $\left(\Gamma^{\prime}\right)^{+}=\Gamma^{+}$.

COROLLARY 2.4. $\Gamma^{+}$is obtained from $\Gamma$ by amalgamating lower branch pairs of edges one by one till it has no such pair.

Similar things hold for $\Gamma^{-}$.

LEMMA 2.5. $\quad \Gamma$ is a chain if and only if both $\Gamma^{+}$and $\Gamma^{-}$are chains.

We define complexity $c(\Gamma)$ of $\Gamma$ as below. Let $v$ be a branch point of $\Gamma^{+}$. Let $e_{1}, \cdots, e_{m}$ be the edges of $\Gamma^{+}$with their initial vertex $v$. Among the graphs obtained by cutting $\Gamma^{+}$at the vertex $v$, let $\Gamma_{i}^{+}$be the component which contains $e_{i}$. Let $n_{i}$ be the number of edges of $\Gamma_{i}^{+}$. Then we define $\omega^{+}\left(\Gamma^{+}, v\right)=\sum_{1 \leq i<j \leq m} n_{i} n_{j}$. Let $\mathcal{B}$ be the set of all the branch points of $\Gamma^{+}$. Then we define $c^{+}(\Gamma)$ by $\sum_{v \in \mathcal{B}} \omega^{+}\left(\Gamma^{+}, v\right)$. (For example, for $\Gamma$ in Figure 2, $\omega^{+}\left(\Gamma^{+}, v_{1}\right)=1 \times 7=7, \omega^{+}\left(\Gamma^{+}, v_{2}\right)=1 \times 2+1 \times 3+2 \times 3=11, \omega^{+}\left(\Gamma^{+}, v_{3}\right)=1 \times 1=1$, and hence $c^{+}(\Gamma)=7+11+1=19$.) Clearly, $c^{+}(\Gamma)=0$ if and only if $\Gamma^{+}$is a chain.

Similarly we define $\omega^{-}\left(\Gamma^{-}, v\right)$ and $c^{-}(\Gamma)$, and set $c(\Gamma)=c^{+}(\Gamma)+c^{-}(\Gamma)$. By Lemma $2.5 c(\Gamma)=0$ if and only if $\Gamma$ is a chain.

LEMMA 2.6. Let $\Gamma^{\prime}$ be a graph obtained from $\Gamma$ by an elementary transformation on a pair of branch edges. Then $c\left(\Gamma^{\prime}\right)=c(\Gamma)-1$.

The next is the main result, and follows immediately from the above lemma.

THEOREM 2.7. $c(\Gamma)$ is equal to the number of elementary transformations for deforming $\Gamma$ to a chain. This number does not depend on the sequence of transformations.

REMARK 2.8. Let $n^{+}$be the number of the edges of $\Gamma^{+}$. Then $c^{+}(\Gamma)=$ $\frac{\left(n^{+}+1\right)\left(n^{+}+2\right)}{2}-\chi\left(\Gamma^{+}\right)$by Remark 4-4 and Corollary 4-5 in [2] and Theorem 2.7 in this paper. (Note that $\Gamma^{+}$satisfies the property (P1) in Remark 4-4.) However, $c^{+}(\Gamma)$ is a little easier to calculate than $\chi\left(\Gamma^{+}\right)$.

COROLLARY 2.9. If a sequence of elementary operations deforms a link diagram $D$ to a closed braid, then the number of the operations is $c(\Gamma)$.

\section{Proofs}

Proof. We prove Lemma 2.3. There is a natural continuous map $\rho: \Gamma \rightarrow \Gamma^{\prime}$, which keeps the heights of the vertices.

First, we prove that a pair of edges $e_{1}, e_{2}$ of $\Gamma$, which are amalgamated in $\Gamma^{+}$, are amalgamated also in $\left(\Gamma^{\prime}\right)^{+}$. If they are amalgamated in $\Gamma^{\prime}$, then we are done. We can assume they are not amalgamated in $\Gamma^{\prime}$. In $\Gamma$ there is a path $\lambda$ connecting the terminal points $v_{1}, v_{2}$ of $e_{1}$ and $e_{2}$ with all its vertices not lower than $h\left(v_{1}\right)=h\left(v_{2}\right)$. The image $\rho(\lambda)$ is connected, 


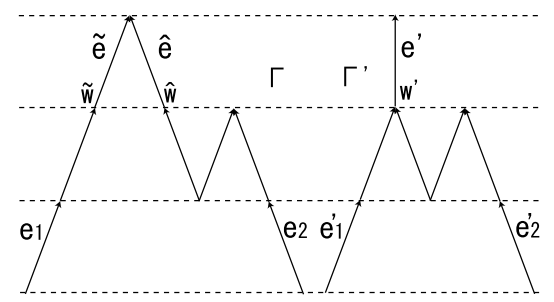

FIGURE 4. $\tilde{e}$ and $\hat{e}$ are amalgamated to form $e^{\prime}$.

and contains $\rho\left(v_{1}\right)$ and $\rho\left(v_{2}\right)$. Hence $\rho(\lambda)$ contains a path $\gamma$ connecting $\rho\left(v_{1}\right)$ and $\rho\left(v_{2}\right)$ in $\Gamma^{\prime}$. (The path is possibly a single point.) No vertex of $\gamma$ is lower than $h\left(\rho\left(v_{1}\right)\right)=h\left(\rho\left(v_{2}\right)\right)$ because $\gamma \subset \rho(\lambda)$. Hence $\rho\left(e_{1}\right)$ and $\rho\left(e_{2}\right)$ are also amalgamated in $\left(\Gamma^{\prime}\right)^{+}$.

Let $\tilde{e}$ and $\hat{e}$ be the lower branch pair of edges in $\Gamma$, which are to be amalgamated to an edge $e^{\prime}$ to form $\Gamma^{\prime}$. Then they share their terminal vertex, and hence are amalgamated in $\Gamma^{+}$. See Figure 4. We prove that a pair of edges $e_{1}^{\prime} e_{2}^{\prime}$ of $\Gamma^{\prime}$, which are amalgamated in $\left(\Gamma^{\prime}\right)^{+}$, are amalgamated also in $\Gamma^{+}$. In $\Gamma^{\prime}$ there is a path $\lambda^{\prime}$ connecting the terminal points $v_{1}^{\prime}, v_{2}^{\prime}$ of $e_{1}^{\prime}$ and $e_{2}^{\prime}$ with all its vertices not lower than $h\left(v_{1}^{\prime}\right)=h\left(v_{2}^{\prime}\right)$. If the preimage $\rho^{-1}\left(\lambda^{\prime}\right)$ is connected, then a similar argument as in the previous paragraph will do. Suppose that $\rho^{-1}\left(\lambda^{\prime}\right)$ is disconnected. Then $\lambda^{\prime}$ contains the initial point $w^{\prime}$ of the edge $e^{\prime}$, and does not contain the edge $e^{\prime}$. The preimage $\rho^{-1}\left(w^{\prime}\right)$ is the union of the two initial vertices $\tilde{w}, \hat{w}$ of $\tilde{e}, \hat{e} \cdot \rho^{-1}\left(\lambda^{\prime}\right)$ consists of two disjoint paths, one of which contains $\tilde{w}$, and the other $\hat{w}$. Hence $\rho^{-1}\left(\lambda^{\prime}\right) \cup \tilde{e} \cup \hat{e}$ is a path connecting $\rho^{-1}\left(v_{1}^{\prime}\right)$ and $\rho^{-1}\left(v_{2}^{\prime}\right)$. Its vertices are not lower than $h\left(\rho^{-1}\left(v_{1}^{\prime}\right)\right)=h\left(\rho^{-1}\left(v_{2}^{\prime}\right)\right)$. Thus $\rho^{-1}\left(e_{1}^{\prime}\right)$ and $\rho^{-1}\left(e_{2}^{\prime}\right)$ are amalgamated in $\Gamma^{+}$.

Proof. We prove Corollary 2.4. Let $\Gamma=\Gamma_{0}, \Gamma_{1}, \cdots, \Gamma_{m}$ be a sequence of trees such that $\Gamma_{i+1}$ is obtained from $\Gamma_{i}$ by amalgamating a lower branch pair of edges and that $\Gamma_{m}$ has no lower branch point. (Every sequence terminates within finite length because amalgamation decreases the number of edges by one and because $\Gamma$ has only finitely many edges.) By Lemma $2.3 \Gamma^{+}=\Gamma_{1}^{+}=\cdots=\Gamma_{m}^{+}$. Since $\Gamma_{m}$ has no lower branch pair of edges, $\Gamma_{m}^{+}=\Gamma_{m}$. Thus $\Gamma^{+}=\Gamma_{m}$.

PROOF. We prove Lemma 2.5 .

"Only if part" is very clear. We consider "if" part.

For the original graph $\Gamma$ and a height $h$ we define $\mu^{+}(\Gamma, h)$ by (the number of edges with initial vertices of height $h$ ) - (the number of such vertices of height $h$ ). We do not count in $\mu^{+}$the vertices which are not initial for any edge. Then, $\mu^{+}(\Gamma, h)=0$ for all $h \in \mathbf{Z}$ if and only if $\Gamma$ has no upper branch point.

Claim. $\mu^{+}(\Gamma, h)=\mu^{+}\left(\Gamma^{+}, h\right)$ for all $h \in \mathbf{Z}$.

PROOF. By Corollary $2.4 \Gamma^{+}$can be obtained from $\Gamma$ by a sequence of amalgamation operations of lower branch pair of edges. It is enough to show that a single operation does 
not change the value of $\mu^{+}$for all $h \in \mathbf{Z}$. In fact, this does not change the numbers of the vertices and the edges counted in $\mu^{+}$at the height other than the level of the initial vertices of the lower branch pair of edges. There both the number of the vertices and that of the edges decrease by one, and hence the value of $\mu^{+}$does not change. This completes the proof of Claim.

If $\Gamma^{+}$is a chain, then $\mu^{+}\left(\Gamma^{+}, h\right)=0$, and hence $\mu^{+}(\Gamma, h)=0$ for all $h \in \mathbf{Z}$. This implies that $\Gamma$ has no upper branch point.

A similar argument shows if $\Gamma^{-}$is a chain, then $\Gamma$ has no lower branch point.

Thus, if both $\Gamma^{+}$and $\Gamma^{-}$are chain, then $\Gamma$ has no branch point, and hence is a chain.

Proof. We prove Lemma 2.6. We will prove $c^{+}\left(\Gamma^{\prime}\right)=c^{+}(\Gamma)-1$ and $c^{-}\left(\Gamma^{\prime}\right)=$ $c^{-}(\Gamma)$ when $\Gamma^{\prime}$ is obtained from $\Gamma$ by an elemantary transformation on an upper branch pair of edges. Similar arguments will do for the case of a lower branch pair of edges.

First, we prove $c^{-}\left(\Gamma^{\prime}\right)=c^{-}(\Gamma)$. Let $e_{1}$ and $e_{2}$ be the upper branch pair of edges of $\Gamma$. Amalgamating them to an edge $e^{\prime}$ we obtain a tree $\tilde{\Gamma}$. To form $\Gamma^{\prime}$, we add a new edge $\theta$ to $\tilde{\Gamma}$ so that its initial vertex is the terminal vertex of $e^{\prime}$ and that its terminal vertex is isolated. By Lemma $2.3 \Gamma^{-}=\tilde{\Gamma}^{-}$. Hence $c^{-}(\Gamma)=c^{-}(\tilde{\Gamma})$. If $\theta$ is amalgamated with some edge in $\Gamma^{\prime-}$ when we form $\Gamma^{\prime-}$ from $\Gamma^{\prime}$, then $\tilde{\Gamma}^{-}=\Gamma^{\prime-}$, and hence $c^{-}(\Gamma)=c^{-}\left(\Gamma^{\prime}\right)$. If $\theta$ is not amalgamated with any edge in $\Gamma^{\prime-}$, then $\Gamma^{\prime-}$ is obtained from $\tilde{\Gamma}^{-}$by adding the edge $\theta$ so that its initial vertex is the terminal vertex of $e^{\prime}$. This does not affect $c^{-}(\tilde{\Gamma})$, which means $c^{-}(\tilde{\Gamma})=c^{-}\left(\Gamma^{\prime}\right)$.

Now we prove $c^{+}\left(\Gamma^{\prime}\right)=c^{+}(\Gamma)-1$. Let $e_{1}, e_{2}, e^{\prime}, \tilde{\Gamma}, \theta$ and $\Gamma^{\prime}$ be as above, and $v$ the common initial vertex of $e_{1}$ and $e_{2}$.

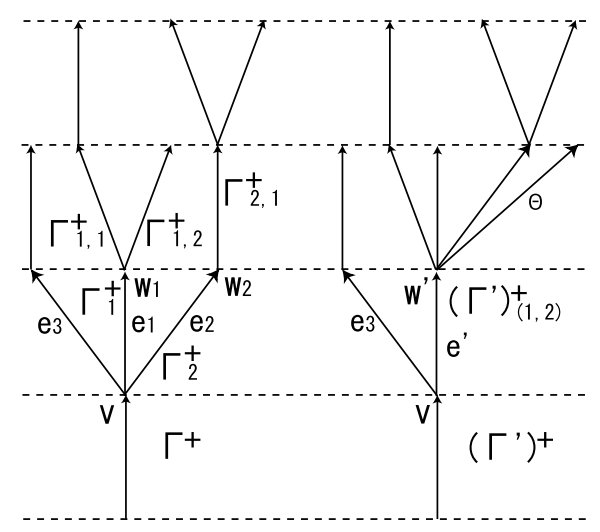

FIGURE 5. Elementary transformation. 
ClAIM. An elementary operation on an upper branch pair in $\Gamma$ causes an elementary operation on the corresponding edges in $\Gamma^{+}$. That is, $\left(\Gamma^{\prime}\right)^{+}$can be obtained from $\Gamma^{+}$by an elementary operation.

Proof. The edges $e_{1}$ and $e_{2}$ are not amalgamated in $\Gamma^{+}$. (If they were, then $\Gamma$ contains a path $\lambda$ connecting their terminal vertices with all its vertices higher than $h(v)$. Hence $\lambda \cup$ $e_{1} \cup e_{2}$ would be a cycle in the tree $\Gamma$.) They are to be amalgamated when we form $\left(\Gamma^{\prime}\right)^{+}$ from $\Gamma^{+}$.

In $\left(\Gamma^{\prime}\right)^{+}$the edge $\theta$ is not amalgamated with another edge of $\Gamma^{\prime}$ because its terminal vertex is isolated. Hence we must add $\theta$ to $\Gamma^{+}$when we form $\left(\Gamma^{\prime}\right)^{+}$.

For $\{i, j\}=\{1,2\}, e_{i}$ and another edge $e$ of $\Gamma^{+}$are amalgamated in $\left(\Gamma^{\prime}\right)^{+}$if and only if $e$ is amalgamated with either $e_{i}$ or $e_{j}$ in $\Gamma^{+}$. In the latter case, when we form $\left(\Gamma^{\prime}\right)^{+}$from $\Gamma^{+}$, the edges $e_{i}$ and $e$ are to be amalgamated, which is realized by amalgamating $e_{1}$ and $e_{2}$.

Besides them, a pair of edges of $\Gamma$ is amalgamated in $\left(\Gamma^{\prime}\right)^{+}$if and only if it is amalgamated in $\Gamma^{+}$. This completes the proof of Claim.

In $\Gamma^{+}$let $e_{3}, \cdots, e_{m}$ be edges with the initial vertex $v$ other than $e_{1}$ and $e_{2}$. See Figure 5. Among the graphs obtained by cutting $\Gamma^{+}$at the vertex $v$, let $\Gamma_{i}^{+}$be the component which contains $e_{i}$. Let $n_{i}$ be the number of edges of $\Gamma_{i}^{+}$. Then $\omega^{+}\left(\Gamma^{+}, v\right)=\sum_{1 \leq i<j \leq m} n_{i} n_{j}$. By the elementary operation, the edges $e_{1}$ and $e_{2}$ are amalgamated into the edge $e^{\prime}$, so that $\Gamma_{1}^{+}$ and $\Gamma_{2}^{+}$are glued into a graph $\tilde{\Gamma}_{(1,2)}^{+}$. Then $\tilde{\Gamma}_{(1,2)}^{+}$is added the edge $\theta$ to be deformed into a graph $\left(\Gamma^{\prime}\right)_{(1,2)}^{+}$which is a subgraph of $\left(\Gamma^{\prime}\right)^{+}$and has $n_{1}+n_{2}$ edges. Hence $\omega^{+}\left(\left(\Gamma^{\prime}\right)^{+}, v\right)=$ $\left(n_{1}+n_{2}\right)\left(\sum_{3 \leq k \leq m} n_{k}\right)+\sum_{3 \leq i<j \leq m} n_{i} n_{j}$. Thus we have $\omega^{+}\left(\left(\Gamma^{\prime}\right)^{+}, v\right)=\omega^{+}\left(\Gamma^{+}, v\right)-n_{1} n_{2}$. The terminal vertices $w_{1}, w_{2}$ of $e_{1}$ and $e_{2}$ are amalgamated to the terminal vertex, say $w^{\prime}$, of $e^{\prime}$ in $\Gamma^{\prime}$. For $i=1$ and 2 , let $\Gamma_{i, 1}^{+}, \cdots, \Gamma_{i, m(i)}^{+}$be the connected components obtained by cutting $\Gamma^{+}$at $w_{i}$ such that $\Gamma_{i, j}^{+}$contains an edge with the initial vertex $w_{i}$. Let $n_{i, j}$ be the number of the edges of $\Gamma_{i, j}^{+}$. Recall that the new edge $\theta$ has the initial vertex $w^{\prime}$ in $\left(\Gamma^{\prime}\right)^{+}$. Hence

$$
\begin{aligned}
\omega^{+}\left(\left(\Gamma^{\prime}\right)^{+}, w^{\prime}\right)-\left(\omega^{+}\left(\Gamma^{+}, w_{1}\right)+\omega^{+}\left(\Gamma^{+}, w_{2}\right)\right) \\
\quad=\left(\sum_{1 \leq i \leq m(1)} \sum_{1 \leq j \leq m(2)} n_{1, i} n_{2, j}\right)+\left(\sum_{1 \leq p \leq m(1)} n_{1, p} \times 1\right)+\left(\sum_{1 \leq q \leq m(2)} n_{2, q} \times 1\right) \\
\quad=\left(\sum_{1 \leq i \leq m(1)} n_{1, i}\right)\left(\sum_{1 \leq j \leq m(2)} n_{2, j}\right)+\left(\sum_{1 \leq p \leq m(1)} n_{1, p} \times 1\right)+\left(\sum_{1 \leq q \leq m(2)} n_{2, q} \times 1\right) \\
=\left(n_{1}-1\right)\left(n_{2}-1\right)+\left(n_{1}-1\right)+\left(n_{2}-1\right) \\
=n_{1} n_{2}-1 .
\end{aligned}
$$

Since $\omega^{+}\left(\left(\Gamma^{\prime}\right)^{+}, x\right)=\omega^{+}\left(\Gamma^{+}, x\right)$ for the other vertex $x$, we obtain $c^{+}\left(\Gamma^{\prime}\right)=c^{+}(\Gamma)-$ 1. 


\section{References}

[ 1 ] J. W. Alexander, A lemma on a system of knotted curves, Proc. Nat. Acad. Sci. USA. 9 (1923), 93-95.

[ 2 ] P. Vogel, Representation of links by braids: A new algorithm, Comment. Math. Helvetici 65 (1990), 104113.

[ 3 ] S. YAMADA, The minimal number of Seifert circles equals the braid index of a link, Invent. Math. 89 (1987), 347-356.

Present Addresses:

CHUICHIRO HAYASHI

Department of Mathematical AND Physical ScienCES,

FACUlTy OF SCIENCE, JAPAN WOMEN'S UNIVERSITY,

MEJIRO-DAI, BUNKYO-KU, TOKYO, 112-8681 JAPAN.

e-mail: hayashic@fc.jwu.ac.jp

HIROKO SAEKI

DEPARTMENT OF MATHEMATICAL AND PHySiCAL SCIENCES,

FACULTY OF SCIENCE, JAPAN WOMEN'S UNIVERSITY,

MEJIRO-DAI, BUNKYO-KU, TOKYO, 112-8681 JAPAN. 